Journal of Case Reports 2017;7(4):404-407

\title{
Ischium: A Rare Location for Osteoid Osteoma
}

\author{
Deepak Kumar, Lokesh Kumar SN \\ Department of Orthopaedics, Postgraduate Institute of Medical Education and Research, Chandigarh-160012, India.
}

\begin{abstract}
Corresponding Author:
Dr. Lokesh Kumar SN

Email: hi1loke@gmail.com

This is an Open Access article distributed under the terms of the Creative Commons Attribution License (creativecommons.org/ licenses/by/3.0).
\end{abstract}

Received Accepted

Published

September 25, 2017

November 29, 2017

December 25, 2017

\begin{abstract}
Background: Osteoid osteoma is a benign osteoblastic tumor of unknown etiology constituting $10-12 \%$ of benign bone tumors. Pelvic involvement has been described mostly in acetabulum. Case Report: 42 years old male patient presented with history of fall and pain in right hip region. X-ray showed a well-circumscribed lytic lesion with central rounded radiolucent nidus in the right ischial tuberosity with surrounding bone sclerosis. Conclusion: Osteoblastoma is a distinct benign tumor to be considered as a differential. Ischial tuberosity is a rare location for osteoid osteoma. This unusual location for this tumor combined with altered clinical symptoms leads to late diagnosis.
\end{abstract}

Keywords: Acetabulum, Osteoblastoma, Osteoid Osteoma, Pain, Pelvis, Pelvic Neoplasm.

\section{Introduction}

Osteoid osteoma, word coined by Jaffe, is a benign osteoblastic tumor of unknown etiology [1]. About $10 \%$ to $12 \%$ of benign bone tumors of the bone are osteoid osteomas [1-3]. Osteoid osteomas are more commonly found in the second or third decades of life, but cases have been reported of osteoid osteomas occurring in older patients. There is a predilection for involvement of axial skeleton; especially the lower extremity with half the cases involving the tibia or femur but almost any bone can be involved. Pelvic involvement has been described mostly in acetabulum.

\section{Case Report}

A 42 year old male patient presented to us in our outpatient department with history of fall and pain in right hip region. Patient did not have any history of fever or any preexisting hip pathology. On examination there was pain and tenderness localized over greater trochanter. Patient also gave history of intermittent dull ache around perianal region. Pain used to subside after taking diclofenac
$50 \mathrm{mg}$. At presentation patient didn't have any pain.

Compression of the pelvis did not reproduce the pain. The hip range of motion was full and pain free. Both legs were neurovascularly intact distally with intact sensation to light touch and palpable dorsalis pedis pulses. The patient was able to stand on each leg and walk without exacerbation of the pain.Patient's routine X-ray of pelvis was taken, which showed a well-circumscribed lytic lesion with central rounded radiolucent nidus in the right ischial tuberosity with surrounding bone sclerosis. Advanced imaging of the pelvis was obtained. Computed tomography (CT) showed a radiodense focus within the right ischial tuberosity with a round central radiolucency with no evidence of cortical disruption, periostitis, or osteolysis but with surrounding bone sclerosis which was typical of osteoid osteoma.

Magnetic resonance imaging (MRI) showed no enhancement with gadolinium, no soft-tissue mass and minimal oedema around the ilium lesion on T2-weighted images.All hematological workup 


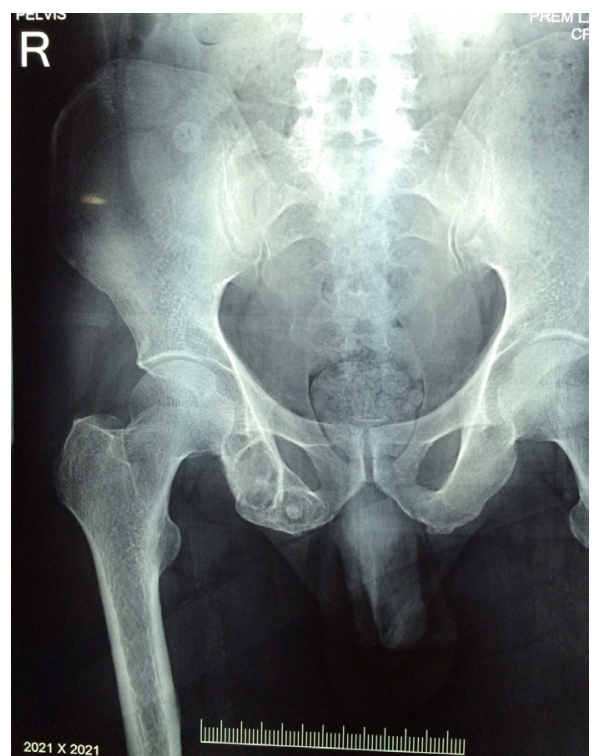

Fig.1: Classical picture of osteoid osteoma.

was performed and were within normal limits. Patient was informed regarding the pathology and its course. No treatment was given in any form except for analgesics. Patient has been kept in close follow up.

\section{Discussion}

The most common bone-producing (i.e. osteoblastic) tumor is osteoid osteoma [4] constituting about $10-12 \%$ of all bone lesions [5-7] and they are relatively benign bone tumors with very rare instances of malignant transformation. Literature reports that as low as only $2.7 \%$ of all osteoid osteoma tumors occur in the pelvis $[6,7]$ with common areas involved include iliac bone and acetabulum. There have been no case reports of osteoid osteoma in ischium as far as to the best of our knowledge and so this becomes a unique case for consideration of such a tumor in an unlikely place. On gross examination, the lesion is usually $<1.5 \mathrm{~cm}$ in diameter and contains a discrete central area of altered density, known as the nidus that is surrounded by dense sclerotic bone [8].

Pelvic osteoid osteoma may present with varied symptoms which may mimic numerous hip pathologies acting as a masquerade. Nocturnal pain

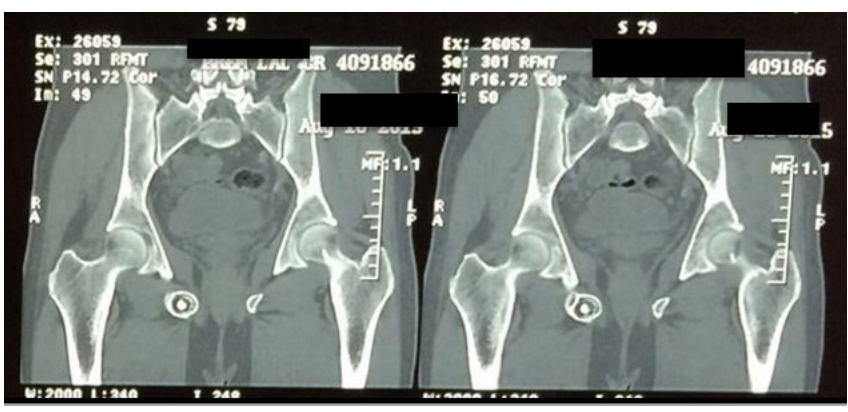

Fig.2: CT scan of pelvis showing central nidus surrounded by radiolucent halo and bony sclerosis surrounding it.

is reported to be the most common symptom of extra-articular osteoid osteoma occurring in about $80 \%$ of patients [9]. The rapid relief with salicylate group of drugs (i.e aspirin, NSAIDs) which is due to inhibition of prostaglandin synthesis which provides transitory but significant pain relief is a very common feature seen in these patients. Delayed diagnosis and incidental asymptomatic finding is common among osteoid osteoma.

Conventional radiographs demonstrating a radiolucent nidus typically less than one $\mathrm{cm}$ in size with a ring of sclerotic bone is considered to be pathognomic of osteoid osteoma, but they might not be visualized in all instances. A radiolucent nidus is best identified with a thin-cut (1-mm) CT scan, which has been proposed as the preferred advanced imaging modality for the diagnosis of osteoid osteoma [10] as CT allows for identification of the nidus, accurate tumor sizing and can facilitate localization for percutaneous treatment. MRI scans also have been used to diagnose the lesion but they have less sensitivity [11]. According to Davis et al, there is a $35 \%$ possibility for a missed diagnosis of osteoid osteoma when diagnosis is solely based on MRI findings [12].

Another highly sensitive diagnostic modality useful for localization and detection of osteoid osteoma is bone scintigraphy. Intraoperative use of portable gamma cameras as radiotracer detectors for localization of the nidus during resection is currently in wide use [13]. Even though 
the test has an increased sensitivity, scintigraphy is still not considered a specific imaging method for accurate diagnosis of osteoid osteoma. Histologically the central nidus characteristically contains tiny osteoid islands that are lined by active osteoblasts. A clear area peripheral to the nidus is always seen because of osteoclastic resorption. There is also reactive bone formation surrounding the lesion due to the high pressure exerted by the tumor on the surrounding normal bone. [14]

Schulman and Dorfman [15] demonstrated abundant nerve fibres located within the matrix of the nidus adjacent to areas rich in arterioles. Pain in osteoid osteoma is due to high levels of prostaglandin synthesis within the nidus of the tumor which have been proven in several studies, which further explains the rapid relief of pain seen on intake of salicylate group of drugs [9].

The classical method of treatment of osteoid osteoma has been surgical excision as the symptoms of osteoid osteoma typically resolve only after a prolonged period [4]. Intra-operative identification of the tumor may be difficult to the naked eye, and recurrence most commonly occurs due to incomplete removal, a standard surgical error in such cases. Resection of weight bearing bone results in functional restriction especially weight bearing, movement of the affected limb and also necessitates the patient for a prolonged hospital stay [16]. Recently, less invasive modalities, including CT-guided excision, arthroscopic excision, drill trepanation with or without ethanol injection, cryoablation, and thermo-ablation with radiofrequency or laser, have evolved, resulting in a decreasing trend for definitive surgical excision.

Osteoblastoma is a distinct benign boneproducing tumor with certain features similar to osteoid osteoma, which should always be considered as a differential. It is of utmost importance to recognize the typical clinical and imaging features of both types of tumor to prevent misdiagnosis, as the differences in management also vary considerably among the two.

\section{Conclusion}

Ischial tuberosity is a rare location for osteoid osteoma. The typical pain pattern may be absent. This unusual location for this tumor combined with altered clinical symptoms leads to late diagnosis along with the possibility of confounding this condition with other pathologies. This brings to light the overwhelming need for careful clinical examination and radiological assessment and also recognition of the importance of this clinical condition. All osteoid osteoma may not require treatment and our patient underwent the same and has been kept under close follow up.

Contributors: DK clinically diagnosed and treated the patient and proof read the article along with critical inputs into the manuscript and LKSN wrote the manuscript along with literary search and was assisting DK in diagnosing and treating the patient. LKSN will act as guarantor. Both authors approved the final version of this manuscript.

Funding: None; Competing interests: None stated.

\section{References}

1. Kransdorf MJ, Stull MA, Gilkey FW, Moser RP Jr. Osteoid osteoma. Radiographs. 1991;11:671-696.

2. Bremer R, Niethard F, Ewerbeck V. Benign bone tumors in the growth years: osteoid osteoma and osteoblastoma. Orthopade.1995;24:24-28.

3. Greenspan A. Benign bone-forming lesions: osteoma, osteoid osteoma, and osteoblastoma: clinical, imaging, pathologic and differential considerations. Skeletal Radiol. 1993;22:485-500.

4. Amanatullah DF, Mallon ZO, Mak WH, Borys D, Tamurian RM. Pelvic osteoid osteoma in a skeletally mature female. Am J Orthop. 2011;40:476-478.

5. Cohen MD, Harrington TM, Ginsburg WW. Osteoid osteoma: 95 cases and a review of the literature. Semin Arthritis Rheum. 1983;12:265-281.

6. Campanacci M, Ruggieri P, Gasbarrini A, Ferraro A, Campanacci L. Osteoid osteoma. Direct visual identification and intralesional excision of the nidus with minimal removal of bone. J Bone Joint Surg Br. 1999;81:814-820.

7. Mounach A, Nouijai A, Ghozlani I, Ghazi M, Achemlal L, Bezza A, et al. Osteoid osteoma of the acetabulum: a case report. Rheumatol Int. 2008;28:601-603.

8. Healey JH, Ghelman B. Osteoid osteoma and osteoblastoma: Current concepts and recent advances. Clin Orthop Relat Res. 1986;204:76-85. 
9. Pikoulas C, Mantzikopoulos G, Thanos L, Passomenos D, Dalamarinis C, Glampedaki-Dagianta K. Unusually located osteoid osteomas. Eur J Radiol. 1995;20:120125.

10. Hosalkar HS, Garg S, Moroz L, Pollack A, Dormans JP. The diagnostic accuracy of MRI versus CT imaging for osteoid osteoma in children. Clin Orthop Relat Res. 2005;433:171-177.

11. Ghanem I. The management of osteoid osteoma: updates and controversies. Curr Opin Pediatr. 2006;18:36-41.

12. Davies M, Cassar-Pullicino VN, Davies AM, McCall IW, Tyrrell PN. The diagnostic accuracy of MR imaging in osteoid osteoma. Skeletal Radiol. 2002;31:559-569.
13. Blaskiewicz DJ, Sure DR, Hedequist DJ, Emans JB, Grant F, Proctor MR. Osteoid osteomas: Intraoperative bone scan-assisted resection. Clinical article. J Neurosurg Pediatr. 2009;4:237-244.

14. Atesok KI, Alman BA, Schemitsch EH, Peyser A, Mankin H. Osteoid osteoma and osteoblastoma. J Am Acad Orthop Surg. 2011;19:678-689.

15. Schulman L, Dorfman HD. Nerve fibers in osteoid osteoma. J Bone Joint Surg Am. 1970;52:1351-1356.

16. Rosenthal DI, Hornicek FJ, Wolfe MW, Jennings LC, Gebhardt MC, Mankin HJ. Percutaneous radiofrequency coagulation of osteoid osteoma compared with operative treatment. J Bone Joint Surg Am. 1998;80:815-821. 